\title{
Análisis de propuestas de Educación Física en casa durante la suspensión de clases por la COVID-19 y orientaciones para su diseño en Educación Primaria Analysis of Physical Education at home proposals during the suspension of face- to-face classes due to COVID-19 and design guidelines in Primary Education
}

\author{
*Iván López-Fernández, **Rafael Burgueño, *Rubén Espejo García, *Francisco Javier Gil-Espinosa
} *Universidad de Málaga (España), **Universidad Isabel I (España)

\begin{abstract}
Resumen. El sistema educativo está tratando de responder de manera eficaz a los retos y cambios en materia educativa provocados por la COVID-19. El proceso de transición de una enseñanza predominantemente presencial a una virtual ha supuesto un esfuerzo considerable para el profesorado de Educación Física (EF) con la finalidad de adaptar el proceso de enseñanza-aprendizaje. No hay evidencias de estudios que hayan analizado las iniciativas de EF en casa realizadas por los docentes. Esta investigación tiene como objetivo analizar, desde una perspectiva curricular, diferentes propuestas de EF en casa con la finalidad de conocer sus características, compartir ejemplos de buenas prácticas y ofrecer al profesorado orientaciones útiles que les ayuden a diseñar propuestas de calidad en el futuro. Los resultados evidenciaron que el perfil predominante de actividad fue un ejercicio individual de carácter motriz centrado en el desarrollo de la condición física y presentada como un reto, donde el alumnado repite una secuencia específica de movimientos con ayuda de internet.
\end{abstract}

Palabras clave: Confinamiento, Ejercicio, Currículo, Enseñanza virtual, Metodología.

\begin{abstract}
The education system attempts to effectively respond to the instructional changes and challenges caused by COVID-19. The adaptation process of predominantly face-to-face teaching to virtual one has involved a substantial effort for Physical Education (PE) teachers with the aim of adapting the teaching and learning process. To the best of our knowledge, no studies have analyzed the different at-home PE units done by teachers. This research aimed at examining, from a curricular perspective, distinct at-home PE proposals in order to ascertain their characteristics, share instances of good teaching practices, and provide teachers with useful guidelines to help them design quality proposals in the future. The results evidenced that the predominant activity profile was an individual motor exercise focused on physical fitness and introduced as a challenge, in which students repeat a specific movement sequence with the aid of the internet.
\end{abstract}

Keywords: Lockdown, Exercise, Curriculum, Online teaching, Methodology.

\section{Introducción}

El mundo en su conjunto se encuentra actualmente enfrentándose a una situación de pandemia provocada por el virus SARS-CoV-2, que afecta a la vida diaria de toda la población (Jeong \& So, 2020). Desde que la Organización Mundial de la Salud declarase el 11 de marzo de 2020 la pandemia por la COVID-19, una serie de medidas, como el evitar las actividades presenciales y el compromiso con la distancia social, se han convertido en una parte de nuestra vida cotidiana (Jeong \& So, 2020). De hecho, esta pandemia también ha provocado cambios en los contextos educativos de la mayoría de los países occidentales, en la medida que ha sido la primera vez que los centros educativos interrumpieron el transcurso habitual del año académico en algunos países (Varea \& González-Calvo, 2020), mientras que en otros

Fecha recepción: 31-03-21. Fecha de aceptación: 06-06-21

Iván López-Fernández

ivanl@uma.es países los centros educativos no pudieron iniciar el curso (Jeong \& So, 2020). En España, ante esta situación inesperada, el sistema educativo está tratando de dar una respuesta eficiente a los urgentes cambios en materia educativa provocados por la COVID-19, llevando a cabo adaptaciones de las programaciones curriculares junto con el desarrollo de nuevos enfoques de enseñanza basados en ambientes virtuales (Cotino-Hueso, 2020).

En el caso particular de la EF, esta asignatura adquiere un papel clave dentro del ámbito educativo a la hora de favorecer, a través del movimiento, el desarrollo integral del alumnado y, a su vez, promocionar la actividad física relacionada con la salud (American College of Sports Medicine, 2020). Dado el inherente carácter tanto práctico como vivencial que caracteriza a la EF (Fernández-Río, 2020), el proceso de adaptación de la clase presencial a la clase virtual ha supuesto un esfuerzo considerable para el profesorado de EF en cuanto a la adaptación de contenidos, estrategias de comunicación o evaluación (Fuentes-Nieto et al., 2020; GilEspinosa, 2020). La mayoría del profesorado de EF muestra actitudes positivas hacia la incorporación de las Tec- 
nologías de la Información y de la Comunicación (TIC) en el aula, al ser entendidas como herramientas facilitadoras del aprendizaje (Díaz-Barahona et al., 2019). El confinamiento ha llevado a una integración de las TIC de modo obligatorio e inmediato para abordar el proceso de enseñanza y aprendizaje por parte del profesorado de EF (Baena-Morales et al., 2021; Gil-Espinosa, 2020).

El uso de las TIC por parte del profesorado de EF ha permitido continuar con las clases de EF durante el confinamiento por medio de una enseñanza virtual (Beard \& Konukman, 2020; González-Rivas et al., 2021). Esto se ha traducido en una proliferación de diferentes propuestas curriculares para seguir realizando EF desde la casa (Burgueño et al., 2020, 2021). El desarrollo de tales propuestas basadas en las TIC ha supuesto también una oportunidad a la hora de generar nuevos recursos didácticos que podrían seguir siendo de utilidad para el profesorado de EF cuando las clases sean exclusivamente presenciales, o bien, combinen la modalidad tanto presencial como virtual (Calderón et al., 2020).

En relación con las propuestas curriculares desarrolladas para la EF en casa, estas ofrecen oportunidades no sólo para la ocupación activa y constructiva del tiempo libre dentro de casa, sino que también pueden contribuir a cumplir con los niveles diarios de actividad física relacionada con la salud para niños (Burgueño et al., 2020, 2021). Estas propuestas de EF ofrecen además alternativas saludables en un entorno donde pasan mucho tiempo y tienen más fácil optar por ser sedentarios. Por otro lado, este tipo de actividad física puede ser desarrollada por los niños de manera más autónoma y sin depender de la voluntad o las posibilidades económicas o de tiempo de las familias, como ocurre con otras actividades físicas extraescolares.

Por todo ello, las propuestas de EF en casa no sólo tienen sentido en casos extremos, como el de la crisis sanitaria derivada de la COVID-19, sino que su relevancia puede beneficiar de manera significativa la educación y la salud de la población escolar en otros escenarios. Hasta el momento, no tenemos conocimiento de estudios que hayan hecho un análisis sobre estas iniciativas de práctica de EF en casa. Este estudio exploratorio no pretende hacer una revisión sistemática de las propuestas realizadas, pero sí analizar desde una perspectiva curricular algunas de ellas, conocer sus características, compartir ejemplos de buenas prácticas y ofrecer orientaciones útiles que ayuden a diseñar propuestas de EF en casa de calidad y a afrontar situaciones de este tipo en el futuro.

\section{Metodología}

\section{Estrategias de búsqueda}

Durante la crisis sanitaria de la COVID-19, internet se ha convertido en un espacio de colaboración y cooperación en el que se han multiplicado las interacciones entre docentes y discentes. La mayor parte de la información se ha enviado al alumnado a través de plataformas de acceso restringido (por ejemplo, Google Classroom o ClassDojo), lo que dificulta la selección de una muestra aleatoria y representativa de colegios. Afortunadamente, muchos docentes han compartido sus propuestas, haciéndolas accesibles no sólo a su alumnado, sino también a otros compañeros docentes y a cualquier persona interesada. El principal canal utilizado han sido los blogs educativos, que son herramientas web 2.0 cuyo objetivo es publicar y compartir contenidos educativos, contribuyendo a que los contenidos estén abiertos a todas las personas y los aprendizajes tengan un carácter más flexible, interactivo y dinámico (Marín Díaz et al., 2020). En el área de EF, los blogs también han sido utilizados con relativa frecuencia por el profesorado como herramienta didáctica para conseguir objetivos educativos (López-Postigo et al., 2021) y se han convertido, durante la pandemia por la COVID-19, en una herramienta básica para la comunicación de actividades, comentarios, noticias, propuestas y recomendaciones (Sierra-Díaz et al., 2021).

En primer lugar, se ha realizado una búsqueda de blogs específicos del área de EF, utilizando el motor de búsqueda de Google e introduciendo las palabras clave «blog» $\mathrm{y}$ «educación física». Se han obtenido más de 8 millones de resultados (8230000 resultados), de los que se han consultado los 100 primeros. Este primer filtro, basado en la clasificación de los resultados, se fundamenta en las propiedades del algoritmo de búsqueda de Google, que incluye, entre otras estrategias para ordenar los resultados, una fórmula denominada «PageRank», que contribuye a organizar los resultados en base a la cantidad de sitios que redirigen a los usuarios a la misma (Wakabayashi, 2018). Google también utiliza criterios adicionales de clasificación, como la frecuencia y en qué lugar de una página específica aparecen las palabras clave que se buscan, la fecha de creación del sitio y la ubicación de la persona que hace la búsqueda (Rovira et al., 2018; Wakabayashi, 2018). Esto ha permitido centrar la búsqueda en un número de resultados relevante y viable.

Tras eliminar seis registros repetidos y cinco que no eran blogs, sino artículos de revistas académicas, se apli- 
caron los siguientes criterios de selección de blogs:

1. Su temática principal es la EF.

2. Orientados a la educación primaria (alumnado de 6 a 12 años).

3. Tienen como mínimo una entrada con una propuesta de EF en casa durante el periodo de suspensión de las clases presenciales.

En base a los criterios indicados, se han seleccionado 21 blogs. Dentro de cada blog seleccionado, se han buscado propuestas de EF para hacer en casa en las entradas al blog comprendidas entre el lunes 16 de marzo de 2020 y el viernes 24 de abril de 2020, habiéndose localizado 65 propuestas. Estas dos fechas coinciden con el inicio de la suspensión de las clases presenciales y el inicio de la posibilidad de salir a la calle, un periodo que contempla 6 semanas en las que las medidas de confinamiento no han permitido al alumnado salir de casa en España. Por su parte, los criterios de selección de las propuestas de EF en casa han sido:

1. El contenido principal de la propuesta es sobre EF.

2. Están dirigidas a alumnado de educación primaria (alumnado de 6 a 12 años) que cursa sus estudios en el sistema educativo vigente en España.

3. El contenido es abierto y accesible de manera gratuita, pudiendo ser consultado por cualquier persona interesada.

4. El autor está identificado.

En base a los criterios expuestos, se seleccionaron

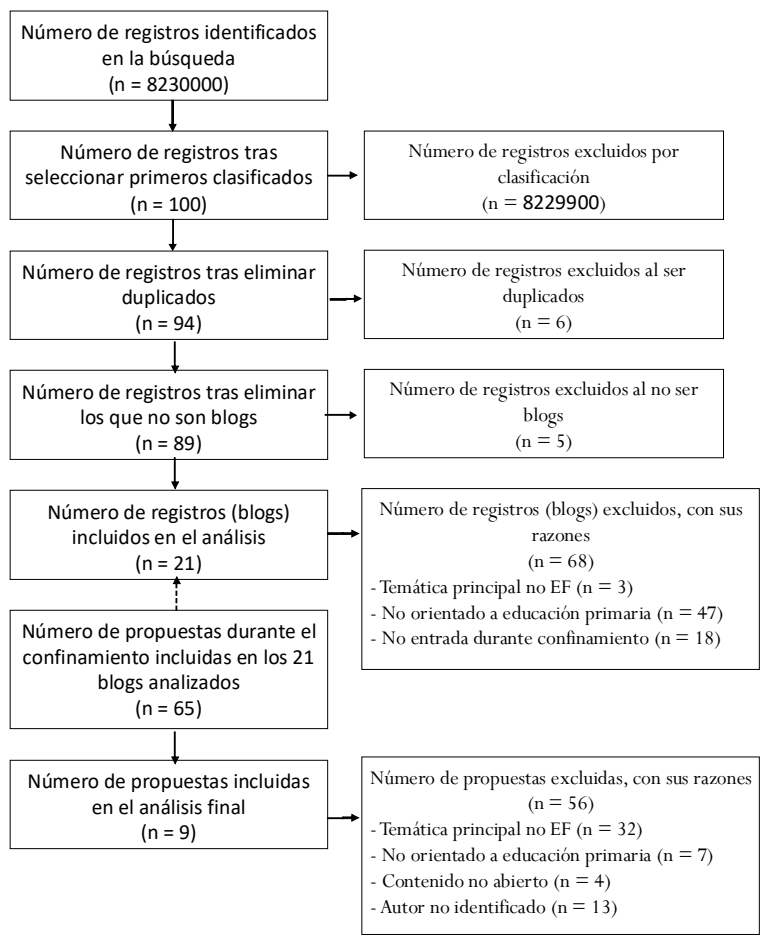

Figura 1. Diagrama de flujo del proceso de selección de blogs y propuestas de Educación Física en casa. Adaptado de Moher et al. (2009) finalmente nueve de las 65 propuestas de EF en casa consultadas. En la Figura 1, se presenta un diagrama de flujo, adaptado de Moher et al. (2009), de los registros obtenidos en cada fase, en el que se especifican: a) el número total de blogs consultados y el número de blogs rechazados sobre cada criterio de selección; b) el número de propuestas de EF en casa (incluidas en cada uno de los blogs finalmente seleccionados) consultadas, así como el número de propuestas de EF en casa rechazadas en base a cada criterio de selección.

En la revisión participaron los cuatro autores del estudio, que aplicaron los criterios de selección de blogs y propuestas de manera independiente. En los casos en los que no existía coincidencia tras cruzar los resultados, la decisión sobre la exclusión o no del registro se tomó de manera consensuada.

\section{Análisis de la información: análisis curricular}

Tomando de referencia el currículum de EF de educación primaria (Real Decreto 126/2014), se han analizado diferentes elementos curriculares, como las competencias, objetivos, evaluación, situaciones motrices, metodología y material de las propuestas de EF en casa.

Se ha valorado la contribución de las propuestas de EF en casa a la consecución de las competencias previstas en el currículum:

- Comunicación lingüística (CCL).

- Competencia matemática y competencias básicas en ciencia y tecnología (CMCT).

- Competencia digital (CD).

- Aprender a aprender (CAA).

- Competencias sociales y cívicas (CSC).

- Sentido de iniciativa y espíritu emprendedor (SIEP).

- Conciencia y expresiones culturales (CEC).

También se ha valorado la aportación de las propuestas a los objetivos, entendidos como referentes relativos a los logros que el alumnado debe obtener al finalizar la educación primaria, así como las características de la evaluación empleada para valorar el grado de adquisición de las competencias y de consecución de los objetivos.

Igualmente, se ha analizado el tipo de situaciones motrices alrededor de las cuales pueden estructurarse los elementos curriculares de la programación de la asignatura de EF que contenían dichas propuestas:

Acciones motrices individuales en entornos estables (AMIEE).

- Acciones motrices en situaciones de oposición (AMSO). 
Acciones motrices en situaciones de cooperación, con o sin oposición (AMSC).

- Acciones motrices en situaciones de adaptación al entorno físico (AMSAEF).

- Acciones motrices de índole artística o de expresión (AMSAE).

Finalmente, se ha examinado la metodología didáctica en cuanto a estrategias, procedimientos $y$, acciones organizadas y planificadas, de manera intencionada, con la finalidad de facilitar el aprendizaje del alumnado y la adquisición de los objetivos planteados. En este sentido, se ha evaluado el tipo de material necesario para desarrollar las actividades.

\section{Resultados y discusión}

En la Tabla 1 se muestran los nueve blogs que fueron incluidos en este estudio y las principales propuestas seleccionadas de acuerdo con los criterios descritos en el apartado anterior. A continuación, la Tabla 2 recoge el análisis curricular de las actividades propuestas, contemplando las competencias clave, objetivos generales de etapa, criterios de evaluación, situaciones motrices, tipos de organización y material necesario para cada una de las propuestas de EF incluidas en este trabajo.

\section{Competencias del currículo}

Las propuestas planteadas contribuyen a la adquisi- ción de todas las competencias previstas en el currículo, si bien la competencia matemática, en ciencias y tecnología parece que recibe menor atención. Igualmente, el trabajo de la competencia lingüística oral y escrita es limitada; el hecho de que algunos de los recursos, como videos de canciones de internet, se encuentren en inglés, facilita la mejora del aprendizaje de una lengua extranjera.

En cambio, se observa que la ausencia de clases presenciales ha potenciado de manera sustancial el desarrollo de la competencia digital. Esta percepción sobre el desarrollo en el alumnado de habilidades en TIC se ha puesto también de manifiesto en un estudio previo, en el que se analizaba la experiencia del profesorado de EF en sus clases durante el confinamiento por la COVID19 en México (González-Rivas et al., 2021). El alumnado utiliza las nuevas tecnologías, desde el momento en el que recibe la información inicial de las tareas que tienen que realizar a través de internet, hasta el momento en que tiene que enviar evidencias al profesorado de su ejecución, normalmente a través de videos. Igualmente, es frecuente que las propuestas de EF vayan acompañadas de recomendaciones y consejos que fomentan un uso responsable de dispositivos electrónicos.

Por otro lado, el trabajo autónomo del estudiante es mayor que en las clases convencionales y algunas de las propuestas contemplan tareas de investigación que obligan al estudiante a poner en marcha estrategias de bús-

Tabla 1

\begin{tabular}{|c|c|c|c|c|c|c|c|c|}
\hline \multicolumn{9}{|c|}{ Actividad / Característica } \\
\hline \#WeAreOlympic2020 & \multicolumn{2}{|c|}{ Cristopher/ CEIP La Estrella (Tenerife) } & \multicolumn{3}{|c|}{ https://youtu.be/3-y4YR2jv3c } & \multicolumn{3}{|c|}{ Elegir un personaje y representar video $30^{\prime \prime}$} \\
\hline Monopoly EF Family & \multicolumn{2}{|c|}{$\begin{array}{l}\text { Sergio Santos / } \\
\text { CEIP Clara Campoamor }\end{array}$} & \multicolumn{3}{|c|}{ https://youtu.be/WH1M8Fl L74 } & \multicolumn{3}{|c|}{$\begin{array}{l}\text { Calles con diferentes contenidos (salud, danza, condición física,...) y } \\
\text { diferentes niveles de dificultad y organización }\end{array}$} \\
\hline Efecto vida & \multicolumn{2}{|c|}{ Juan Pavón } & \multicolumn{3}{|c|}{$\begin{array}{l}\text { https://view.genial.ly/5e70f8bbbc60c602d6e8f541/prese } \\
\text { ntation-efecto-vida-10-y-2o }\end{array}$} & \multicolumn{3}{|c|}{$\begin{array}{l}\text { Realizar misiones para combatir virus: minigolf, lectura, investigación (sobre } \\
\text { JJOO), hábitos saludables, juegos populares, nudos, yoga... }\end{array}$} \\
\hline Escape Coronaviroom & \multicolumn{2}{|c|}{ Gabriel Sevillano Ledesma } & \multicolumn{3}{|c|}{ http://gabieducacion.blogspot.com/p/blog-page.html } & \multicolumn{3}{|c|}{$\begin{array}{l}\text { Allbum ART-EF mediante la aplicación Endomondo para completar retos } \\
\text { semanales de distinta índole }\end{array}$} \\
\hline Contracovid-19 & \multicolumn{2}{|c|}{$\begin{array}{l}\text { José Manuel Cenizo Benjumea y Daniel Avilés } \\
\text { Castillo / } \\
\text { CEIP Miguel Rueda (Paradas, Sevilla) }\end{array}$} & \multicolumn{3}{|c|}{$\begin{array}{l}\text { shttps: //my.pcloud.com/publink/show?code =XZLuYMkZ } \\
\text { twec1SxUd2juffuKpYrpCRtuD1Ak }\end{array}$} & \multicolumn{3}{|c|}{$\begin{array}{l}\text { Misiones que implican a toda población. Se consiguen puntos mandando } \\
\text { videos haciendo AF de forma individual, grupal o en colaboración con otros } \\
\text { miembros. Las actividades físicas son de libre elección }\end{array}$} \\
\hline Siempre en forma & \multicolumn{2}{|c|}{$\begin{array}{l}\text { Miguel Angel González Lozano / } \\
\text { CEIP Profesora María Doña (Sevilla) }\end{array}$} & \multicolumn{3}{|c|}{$\begin{array}{l}\text { https: //view.genial.ly/5e7bf4cd2be } 3850 \mathrm{~d} 9 \mathrm{c} 8 \mathrm{ef} 742 / \text { horiz } \\
\text { ontal-infographic-review-ef-en-casa }\end{array}$} & \multicolumn{3}{|c|}{$\begin{array}{l}\text { Diversas propuestas para mantenerse activo, incluyendo tres juegos de mesa } \\
\text { motores con misiones de habilidad, condición física y danza }\end{array}$} \\
\hline Cuida tu cuerpo & \multicolumn{5}{|c|}{$\begin{array}{l}\text { Carlos Chamorro Durán(+PIEFCITOS)/ CEIPhttps://view.genial.ly/5e6b3a48eaeafb0fdd69315b/prese } \\
\text { Cristobal Colón de Villaverde (Madrid) }\end{array}$} & \multicolumn{3}{|c|}{$\begin{array}{l}\text { nDiversas propuestas diarias para mantenerse activos así como } \\
\text { recomendaciones diarias, material autoconstruido, dieta, juegos de mesa, etc. }\end{array}$} \\
\hline Jumangym & \multicolumn{2}{|c|}{ Alejandro Bellerín } & \multicolumn{6}{|c|}{$\begin{array}{l}\text { htps://view.genial.ly/5e7f5a3150a0310d8c26e13e/game } \\
\text {-juego-de-educacion-fisica-en-casa } \\
\text { condición física }\end{array}$} \\
\hline $\begin{array}{l}\text { Coreografía y } \\
\text { cronopuntería }\end{array}$ & \multicolumn{2}{|c|}{ Moisés Moncho } & \multicolumn{6}{|c|}{$\begin{array}{l}\text { https: //u.pcloud.link/publink/show?code }=\text { XZSM4PkZEc } \\
\text { YHAkclq05oaDn3jxz4M74fCzMy }\end{array}$} \\
\hline \multicolumn{9}{|c|}{ Nota: Los enlaces a las páginas web han sido consultados entre el 27 de abril y el 10 de mayo de 2020.} \\
\hline \multicolumn{9}{|l|}{$\begin{array}{l}\text { Tabla } 2 \\
\text { Análisis cu }\end{array}$} \\
\hline Propuesta & & $\mathrm{CC}$ & & $\begin{array}{l}\text { Criterios de } \\
\text { Evaluación }\end{array}$ & Situación motriz & & Organización & Material \\
\hline$\overline{\text { Reto \#WeAreOlympic2 }}$ & 2020 & CAA, CD, SIEP, CEC & & 1,2 & AMSAE & & & Ropa para disfrazarse \\
\hline Monopoly EF Family & & $\mathrm{CCL}, \mathrm{CAA}, \mathrm{CD}, \mathrm{CSC}$ & & $1,2,5$ & AMIEE, AMSAE & & I, PP, PG & Ninguno \\
\hline Efecto vida & & CCL, CMCT, CD, CAA, CSC, SIEP, CE & & $1,2,5,8,12$ & AMIEE, AMSO, AM & MSAE & I, PP, PG & Vasos, globos y material reciclado \\
\hline Escape Coronaviroom & & CCL, CSC, CEC & & $1,2,5$ & AMIEE, AMSO, AM & MSAE & I, PP, PG & Globos \\
\hline Contracovid-19 & & $\mathrm{CD}, \mathrm{CSC}, \mathrm{SIEP}$ & & $1,2,5,13$ & AMIEE, AMSO, AM & MSAE & I, PP, PG & Material variado \\
\hline Siempre en forma & & $\mathrm{CD}, \mathrm{CAA}$ & & $1,2,5$ & AMIEE, AMSO, AM & MSAE & I, PP & Comba, escoba, papel, reciclado... \\
\hline Cuida tu cuerpo & & CCL, CMCT, CD, CAA, CSC, SIEP, CE & & $1,2,5,13$ & AMIEE, AMSO, AM & MSC, AMSAE & I, PP, PG & Reciclado y otro frecuente en hogares \\
\hline Jumangym & & CCL, CMC, CD, CAA, SIEP & & 5,6 & AMIEE, AMSAE & & I, PP & Ninguno \\
\hline Coreografía y cronopun & ntería & CCL, CMC, CD, CAA, SIEP, CEC & & 1,2 & AMIEE, AMSAE & & I & Pelota o papel, cubo o papelera \\
\hline
\end{tabular}


queda, selección y síntesis de la información registrada, lo que contribuye a desarrollar el aprendizaje autónomo (competencia aprender a aprender). Otra cuestión es que todo el alumnado, en general poco habituado a trabajar de manera autónoma, haya podido asumir con eficacia un incremento tan brusco de responsabilidad (Fernández-Río, 2020).

Aunque la mayoría de propuestas relacionadas con la expresión corporal se limitan a la reproducción de bailes disponibles a través de internet, hay ocasiones en las que se otorga más responsabilidad al estudiante, al que se propone la creación de coreografías a partir de una música elegida libremente, desarrollando de esta forma el sentido de la iniciativa. Los contenidos relacionados con la alimentación también fomentan la iniciativa al estimular, por ejemplo, la preparación de recetas en familia o el autocontrol en el consumo de frutas y verduras.

Las manifestaciones artísticas que contemplan algunas propuestas de expresión corporal contribuyen al desarrollo de la conciencia y expresiones culturales, no solo como actores, sino también como espectadores de las actuaciones realizadas por los compañeros, el docente o las observadas en enlaces de internet externos. Las actividades de fitness protagonistas de las propuestas también representan manifestaciones culturales de la sociedad en la que vivimos.

\section{Objetivos de etapa}

Las actividades propuestas han contribuido a la consecución de los objetivos generales de Educación Primaria, destacando el objetivo que hace referencia a la valoración de la higiene y la salud, la aceptación del propio cuerpo y el de los otros, el respeto de las diferencias y la utilización de la EF y el deporte como medios para favorecer el desarrollo personal y social. En este caso, las actividades desarrolladas en el colegio no difieren de las realizadas en casa, en las que este objetivo sigue siendo el protagonista del área de EF. Otro de los objetivos, cuya consecución se ha visto especialmente favorecida, ha sido el relativo a la utilización de representaciones y expresiones artísticas y la iniciación en la construcción de propuestas visuales y audiovisuales. El peso que han tenido los contenidos de expresión corporal en el conjunto de actividades programadas explica la contribución a este último objetivo.

\section{Evaluación}

La evaluación no presencial ha supuesto un reto adicional para los docentes de EF durante el confinamiento por la COVID-19 (Baena-Morales et al., 2021 ; GonzálezRivas et al., 2021). En este caso, el profesorado ha solicitado en la mayoría de ocasiones evidencias de aprendizaje al alumnado a través del envío de fotos y videos. Solo una de las propuestas recurre a la autoevaluación, es decir, a la evaluación por parte del propio alumnado.

Las propuestas analizadas no explicitan normalmente los criterios de evaluación utilizados, si bien las limitaciones que impone la enseñanza a distancia, el tipo de actividades realizadas y las evidencias de aprendizaje empleadas reflejan que los criterios de evaluación protagonistas han sido, básicamente, los tres siguientes:

- Resolver situaciones motrices con diversidad de estímulos y condicionantes espacio-temporales, seleccionando y combinando las habilidades motrices básicas y adaptándolas a las condiciones establecidas de forma eficaz.

- Utilizar los recursos expresivos del cuerpo y el movimiento, de forma estética y creativa, comunicando sensaciones, emociones e ideas.

- Reconocer los efectos del ejercicio físico, la higiene, la alimentación y los hábitos posturales sobre la salud y el bienestar, manifestando una actitud responsable hacia uno mismo.

Por último, es de destacar el compromiso de dos de las propuestas al contemplar como criterio de evaluación la demostración de un comportamiento personal y social responsable.

$\mathrm{Al}$ igual que ha ocurrido en otros países, la ausencia de orientaciones para la evaluación no presencial en este periodo, por parte de las administraciones educativas, ha dificultado esta tarea (Jeong \& So, 2020). Esta falta de orientaciones puede explicar, en parte, que no se hayan identificado más criterios de evaluación en las propuestas analizadas.

\section{Situaciones motrices}

Las acciones motrices predominantes presentes en estas propuestas de EF en casa son las acciones motrices individuales en entornos estables centradas en la preparación física de forma individual, a veces en formato de circuito o como retos independientes a superar. Este dato sintoniza con los resultados de encuestas previas pasadas docentes de EF durante la cuarentena por la COVID-19, en las que insistían en el protagonismo que habían tenido los contenidos relacionados con la condición física y la salud (Baena-Morales et al., 2021; Mercier et al., 2021).

En segundo lugar, se encuentran las acciones motrices individuales de índole artística o de expresión, desta- 
cando los bailes realizados con videos de internet como soporte y, en menor medida, representaciones.

La mayoría de estos tipos de actividades están orientadas para que se puedan practicar de manera individual, pero muchas permiten su adaptación para practicarse por parejas o en pequeños grupos familiares. Las actividades que solicitan o requieren la participación de miembros de la familia en la actividad suplen, en cierta medida, la ausencia de compañeros de clase para jugar. Esto facilita que, en ocasiones, se puedan desarrollar acciones motrices en situaciones de oposición (juegos de uno contra uno) y, más rara vez, en situaciones de cooperación con o sin oposición (juegos tradicionales). Se aprecia cómo la participación de otros miembros de la familia no tiene por qué implicar un cambio en el movimiento, pero la situación motriz es complemente diferente. Llaman la atención algunas ideas muy interesantes para implicar a las familias (como el «maratón en familia») o incluso a comunidades enteras (Contracovid-19). En una coyuntura en la que las actividades de EF adolecen de falta de una vertiente afectiva y social (Jeong \& So, 2020), estas propuestas sugieren que las situaciones motrices no tienen por qué circunscribirse sólo a las individuales, a la vez que cuestionan el encasillamiento del docente de EF en entornos virtuales como mero prescriptor de ejercicio físico para el desarrollo de la condición física, si bien hay que reconocer las limitaciones de la enseñanza a distancia para el desarrollo pleno de todas las acciones motrices en EF (Gil-Ares, 2020).

La ausencia de acciones motrices en situaciones de adaptación al entorno físico entre las propuestas analizadas se justifica porque el hogar, que es el medio en el que se realizan las actividades, genera poca incertidumbre al tener características poco cambiantes. Por las condiciones del confinamiento, no se proponen actividades en el entorno natural o urbano al aire libre. Estos condicionantes justifican la opinión del profesorado que enseña EF con la COVID-19, cuando admite estar muy limitado en cuanto a los contenidos a impartir (Hortigüela-Alcalá et al., 2021).

\section{Metodología del profesorado}

Se observa una gran variedad metodológica, desde propuestas de actividades de preparación física a través de ejercicios estandarizados y repetitivos (mando directo) hasta propuestas que fomentan la autonomía y la creatividad del alumnado. Son más frecuentes el primer tipo de actividades, en las que solo hay que reproducir un modelo físico y en las que la exigencia cognitiva y de toma de decisiones del participante es reducida. Tal es el caso de las numerosas propuestas de baile en la que el discente solo tiene que repetir y memorizar los pasos que se proponen al ritmo de la música en un video.

La gamificación está presente en algunas de las propuestas. Las actividades no se presentan aisladas, sino que hay centros de interés simbólicos sobre los que pivotan las actividades, puntos de unión que les dan sentido (por ejemplo, superar retos o hacer misiones para combatir el coronavirus). Otro ejemplo de gamificación ha sido la utilización de juegos de mesa como recurso, vinculando las casillas del juego a diferentes pruebas motrices. Esta orientación evita que las propuestas se presenten como una sucesión de juegos y ejercicios inconexos sin sentido de continuidad, a la vez que fomentan la motivación del alumnado, incrementando su potencial educativo (López-Fernández \& Malavé, 2009). La dimensión lúdica contribuye también a mitigar la monotonía derivada del entorno en el que desarrolla la EF (la propia casa) y de las limitaciones del tipo de acciones motrices propuestas en las experiencias de EF en casa (Jeong \& So, 2020). De acuerdo con los resultados de una encuesta en la que participaron más de 4000 docentes estadounidenses, conseguir que el alumnado valore y se divierta haciendo actividad física ha sido uno de los objetivos que han priorizado durante la enseñanza no presencial de EF (Mercier et al., 2021).

La mayoría de las actividades no son de producción propia, es decir, no son creadas por el docente de EF, sino que su labor se «limita» a seleccionar información ya existente en la red. En este sentido, el docente se convierte en un gestor de contenidos digitales o intermediario del conocimiento que busca, selecciona, organiza, adapta, reúne, explica y comparte los recursos que más se adecúan a su contexto educativo. Este nuevo rol del docente, cada vez más necesario en la sociedad de la información en la que vivimos (Godoy-Rodríguez, 2018), requiere un esfuerzo y una inversión de tiempo considerable, además de contar con fuentes de información de calidad, estrategias de búsqueda eficientes, criterios de selección adecuados, recursos organizativos, conocimiento profundo del entorno educativo al que se dirige la propuesta y dominio de aplicaciones informáticas para compartirlo con el alumnado.

\section{Material}

Las propuestas analizadas no utilizan mucho material y, cuando lo hacen, este suele ser sencillo, barato y accesible: palos de escoba, dados, rollos de papel... La 
elaboración de material para la práctica de actividad física reutilizando o reciclando material de desecho es frecuente en muchas propuestas. La inexistencia de material específico deportivo, habitual en los gimnasios de los centros educativos, no parece ser un obstáculo para el desarrollo de las actividades, que se adaptan a la realidad de los hogares.

No obstante, hay que destacar que el canal de información utilizado para comunicarse es digital, lo que requiere contar con un dispositivo electrónico con conexión a internet. En este sentido, hay propuestas que son menos dependientes de los medios electrónicos y utilizan un texto (generalmente en formato PDF) como único soporte para desarrollar la actividad, mientras que otros implican una conexión permanente para la elaboración de las tareas (con tráfico de datos elevado, como videos). Algunos docentes presentan alternativas que reducen las exigencias tecnológicas para el envío de evidencias para la evaluación; por ejemplo, sugiriendo que graben los videos en un pendrive que se entregaría al final de la evaluación. Este tipo de adaptaciones son relevantes para reducir la brecha digital e intentar asegurar la igualdad de oportunidades de aprendizaje, que se ha visto comprometida durante el confinamiento en casa (Bonal \& Gonzalez, 2020).

Una desventaja de este tipo de recursos digitales alojados en blogs es la inevitable volatilidad de los enlaces que dan acceso a estos recursos. $\mathrm{Al}$ ser contenidos orientados, en principio, a un periodo concreto, su disponibilidad depende de la decisión que tomen los autores al respecto o de contingencias como el mantenimiento del dominio que alberga el recurso, a diferencia de publicaciones en revistas académicas o en otros soportes más estables. Igualmente, algunos de los enlaces externos que contienen estos recursos educativos también pueden ser retirados, entre otras razones, por cuestiones relacionadas con permisos y derechos de autor.

\section{Implicaciones prácticas para el profesora- do}

Ante el confinamiento del alumnado, al no ser posible una modalidad de docencia presencial, se debería reflexionar sobre la conveniencia de utilizar un modelo no presencial sincrónico o asincrónico, en función de los recursos tecnológicos disponibles y el contexto sociocultural del centro y de las familias.

Como se puede apreciar en los apartados anteriores, el estudio de las propuestas de EF desarrolladas durante el confinamiento del alumnado nos permite conocer ejemplos de buenas prácticas y, a la vez, detectar algunas lagunas o deficiencias. Este conocimiento tiene implicaciones prácticas muy relevantes para el profesorado de $\mathrm{EF}$, puesto que podría ayudar a alcanzar niveles de calidad que faciliten el mejor desarrollo posible de las competencias clave y objetivos. A continuación, se exponen de manera resumida dichas implicaciones prácticas, que se traducen en orientaciones a considerar para el diseño de actividades de EF en casa, agrupadas por temáticas con la finalidad de facilitar la comprensión.

\section{Currículo}

- La EF en casa debe continuar partiendo del marco normativo educativo y no convertirse en una inconexa «prescripción» de ejercicio físico.

- Se deben diseñar actividades en relación a los criterios de evaluación correspondientes.

- Procurar un aprendizaje competencial, trabajando todas las situaciones motrices o bloques de contenidos.

Priorizar los aspectos procedimentales y el desarrollo de la competencia corporal, para la salud y calidad de vida.

\section{Metodología}

- Trabajar fundamentalmente en metodologías innovadoras, productivas, individualizadas y adaptadas al contexto familiar.

- Utilizar la gamificación y plantear retos o desafíos, tanto individuales como colectivos, que contribuyan a aumentar la motivación.

- Avanzar hacia el desarrollo de la autonomía del alumnado, incrementando su toma de decisiones y elección.

Permitir la implicación y participación familiar, si el contexto lo permite.

- Utilizar las TIC para el aprendizaje, motivación, feedback con alumnado y familias. Emplear aplicaciones y redes sociales para enriquecer las propuestas curriculares.

- Trabajar la socialización y relaciones entre el alumnado, haciendo uso de las tecnologías para el aprendizaje y el conocimiento.

- Asegurarse de que la información transmitida respecto a las actividades es suficiente para su correcta ejecución. Ayudarse de recursos como vídeos. Evitar imágenes aisladas que pudiesen llevar a errores.

\section{Actividades de enseñanza-aprendizaje}

- Procurar la participación del alumnado en la pla- 
nificación de dichas actividades y permitir adoptar ciertas decisiones en cuanto a la tipología de estas. Una opción podría ser el planteamiento de diversas actividades a elección, dando la posibilidad de adaptarlas o secuenciarlas según intereses o recursos.

Planificar las actividades contextualizadas y secuenciadas en un conjunto o plan de trabajo, que bien podría ser la unidad didáctica. Evitar actividades inconexas y sin secuencia lógica. Hacer al alumnado conocedor del proceso con una introducción inicial.

- Evitar actividades complejas o cuya ejecución incorrecta pudiese provocar lesiones.

- Aprovechar las actividades y aprendizajes previos de los estudiantes como punto de partida.

- Proponer actividades de relajación y respiración (yoga, masaje en familia, control de la respiración...)

- Proponer actividades de control postural.

- Incluir actividades físicas domésticas (barrer, fregar, tender...)

- Proponer actividades en equipo, de cooperación y colaboración entre el alumnado, utilizando las TIC. La contribución individual al equipo debe ser flexible para adaptarse al contexto personal de cada estudiante.

- En general, se deben proponer actividades flexibles y adaptadas a espacios reducidos.

- Tener en consideración las características y particularidades de cada núcleo familiar. Consideración especial con el alumnado que no tendrá colaboración de adultos para la realización de las actividades.

- Fomentar la implicación familiar en la realización de las actividades. Se pueden incluir variantes que permitan la participación de otros miembros de la familia, pero no hacerlo obligatorio.

\section{Evaluación}

- Diseñar un sistema basado en evidencias de realización y de aprendizaje (fotografías, videos, diario...). Pueden variar según el contenido, madurez, edad del alumnado y permisos familiares.

- Utilizar la auto y coevaluación en las actividades que así lo permitan, en forma de diario personal, lista de control...

- Posibilidad de contrato de aprendizaje con alumnado y familia como parte del proceso de evaluación (firma de los tutores legales como evidencia de realización o aprendizaje...)

- Continua y formativa. Debe existir feedback que ayude a orientar el aprendizaje lo más frecuentemente posible.

- Realizar una puesta en común al final del plan de trabajo o unidad didáctica, haciendo explícitos los aprendizajes.

\section{Transversalidad}

- Avanzar hacia propuestas interdisciplinares e intradisciplinares.

- Aprendizaje lo más globalizado posible.

- Incluir cuestiones relacionadas con la educación para la salud, entendida en todas sus dimensiones.

\section{Atención a la diversidad}

- Diseño que permita la individualización de las actividades, proponiendo variables que posibiliten regular la intensidad, repeticiones, dificultad, utilización de medios disponibles, motivación del alumnado.

- Proponer actividades complementarias y voluntarias que permitan al alumnado incrementar el tiempo de actividad física semanal, avanzando hacia los 60 minutos diarios.

- Flexibilizar los tiempos y momentos de realización y entrega.

\section{Rol docente}

- Procurar un trabajo en equipo tanto con docentes del mismo centro educativo como a través de redes de docentes.

\section{Material y recursos}

- El material necesario debe ser accesible en cualquier hogar.

- Proponer diferentes alternativas y posibilidades en cuanto al material a utilizar.

- Permitir al alumnado el diseño creativo de actividades relacionadas con la unidad didáctica utilizando el material que tenga disponible.

\section{Prevención de riesgos y protección de datos per-} sonales

- Es conveniente explicar al alumnado y familia, los posibles riesgos en la realización de actividad física en casa y en el uso de las nuevas tecnologías.

- Sería conveniente dedicar una introducción general que informe sobre la prevención de riesgos en la realización de actividad física en casa.

- Utilizar las plataformas de comunicación y educativas que el centro educativo tenga autorizadas.

- Introducción inicial general sobre el uso de las TIC, privacidad, control de contraseñas, etc. Al tratarse de una cuestión general, sería conveniente que el centro educativo realizase esta acción de forma conjunta 
para todas las asignaturas y, desde el área de EF, destacar exclusivamente las cuestiones más específicas.

Posteriormente, en cada unidad didáctica, sesión o actividad, advertir específicamente de los posibles riesgos que pudiesen aparecer.

- Asegurarse de que las propuestas de actividades basadas en vídeos o canales de redes sociales son dirigidas o revisadas por profesionales.

Obtener autorización de los tutores legales del alumnado para el uso de enlaces externos que pudiesen contener publicidad, aunque se evitarán lo máximo posible.

Obtener autorización de los tutores legales del alumnado para el envío de imágenes o videos donde aparezca el alumnado, y respetar la normativa vigente sobre protección de datos. En caso de no obtener dicha autorización, será necesario adaptar el proceso de enseñanza-aprendizaje.

\section{Conclusiones}

La suspensión de la actividad docente presencial motivada por la COVID-19 ha supuesto un enorme desafío para el profesorado de EF, que ha tenido que trasladar de manera acelerada la docencia de una asignatura con un marcado carácter práctico del entorno del colegio a un entorno familiar mucho más diverso (tipo de vivienda, disponibilidad de medio tecnológicos, materiales, conocimientos y disponibilidad de los adultos, etc.). La respuesta del profesorado a este reto se ha traducido en multitud de actividades, recursos e ideas para desarrollar el currículo según las características del contexto que rodea a cada estudiante. Si se tuviera que definir de manera breve la tipología de actividades de EF en casa propuestas durante el período de confinamiento por la COVID-19, se podría indicar que el perfil predominante de actividad fue el de un ejercicio motriz e individual centrado en el desarrollo de la condición física y presentado como reto. En este ejercicio, el alumnado, sin una supervisión directa, debía repetir una secuencia específica de movimientos siguiendo el modelo de una imagen o video disponible en internet, siendo realizados a veces al ritmo de la música.

El análisis de algunas de dichas iniciativas realizado en el presente estudio nos permite aprender de la experiencia y aprovecharla para mejorar la formación del profesorado de EF en entornos virtuales. En este sentido, sería conveniente que, partiendo de los criterios de evaluación, se diseñen actividades flexibles, con variables que permitan su adaptación a los diferentes am- bientes familiares, tanto a nivel de espacio físico, como social, cultural y disposición de materiales y recursos. El trabajo en equipo del profesorado y la adaptación a los medios tecnológicos y conocimientos de cada estudiante y su familia cobra una relevancia especial, para evitar riesgos de exclusión educativa por esos motivos.

\section{Agradecimientos}

Agradecemos al profesorado que ha compartido en la red de forma libre sus propuestas de EF en casa durante el confinamiento.

\section{Referencias}

American College of Sports Medicine. (2020). Keeping children active during the coronavirus pandemic. https:/ / www.exerciseismedicine.org/assets / page_documents/EIM_Rx for Health_Keeping Children Active During Coronavirus Pandemic.pdf Baena-Morales, S., López-Morales, J., \& García-Taibo, O. (2021). La intervención docente en educación física durante el periodo de cuarentena por COVID19. Retos, 39, 388-395. https: / / doi.org/10.47197/ retos.v0i39.80089

Beard, J., \& Konukman, F. (2020). Teaching online physical education: The art of connection in the digital classroom. Journal of Physical Education, Recreation \& Dance, 91(7), 49-51. https://doi.org/10.1080/ 07303084.2020 .1785772

Bonal, X., \& Gonzalez, S. (2020). The impact of lockdown on the learning gap: Family and school divisions in times of crisis. International Review of Education, 66, 635-655. https://doi.org/10.1007/ s11159-020-09860-z

Burgueño, R., Bonet-Medina, A., Cerván-Cantón, Á., Espejo, R., Fernández-Berguillo, F. B., Gordo-Ruiz, F., Linares-Martínez, H., Montenegro-Lozano, S., Ordoñez-Tejero, N., \& Vergara-Luque, J. J. (2021). Educación física en casa de calidad. Propuesta de aplicación curricular en educación secundaria obligatoria. Retos, 39, 787-793. https://doi.org/10.47197/ retos.v0i39.78792

Burgueño, R., Espejo, R., López-Fernández, I., \& GilEspinosa, F. J. (2020). Educación física de calidad en casa para niños: Una propuesta de aplicación curricular en educación primaria. Journal of Sport and Health Research, 12(2), 270-287.

Calderón, A., Scanlon, D., MacPhail, A., \& Moody, B. (2020). An integrated blended learning approach for 
physical education teacher education programmes: Teacher educators' and pre-service teachers' experiences. Physical Education and Sport Pedagogy. https: / /doi.org/10.1080/17408989.2020.1823961

Cotino-Hueso, L. (2020). La enseñanza digital en serio y el derecho a la educación en tiempos del coronavirus. Revista de Educación y Derecho, 21, 1-29. https: / / doi.org/10.1344/REYD2020.21.31283

Díaz-Barahona, J., Molina-García, J. , \& Monfort-Pañego, M. (2019). Primary physical education teachers' attitudes and interests towards ICT in the Valencian Community. Retos, 35, 267-272. https: //doi.org/ $10.47197 /$ retos.v0i35.63355

Fernández-Río, J. (2020). Apuntes metodológicos para una educación física post-COVID-19. EmásF: Revista Digital de Educación Física, 66, 67-75.

Fuentes-Nieto, T., Jiménez Herranz, B., López Pastor, V. M., Regidor Sanz, S., Gómez, Á., Ruano Herranz, C., Sáez Laguna, J., Ramos Benito, M. A., Yugueros Martín, Á., \& Magaña Salamanca, E. (2020). Educación física en tiempos de coronavirus y confinamiento: Análisis desde la experiencia. EmásF:Revista Digital de Educación Física, 65, 151-181.

Gil-Ares, J. (2020). «La «nueva normalidad» en educación física. EmásF: Revista Digital de Educación Física, 66, 5-9.

Gil-Espinosa, F. J. (2020). La covid-19: Una carrera de fondo para avanzar en educación física. Journal of Physical Education and Human Movement, 2(2), 1-5. h t t p s : / / d o i . org/ 10.24310 / JPEHMjpehmjpehm.v2i29849

Godoy-Rodríguez, C. (2018). La curación de contenidos digitales. Competencia indispensable para los docentes del siglo XXI. Delectus, 1(1), 51-65.

González-Rivas, R.A., Gastélum-Cuadras, G., Velducea, W., Bernabé González Bustos, J., \& Domínguez Esparza, S. (2021). Análisis de la experiencia docente en clases de educación física durante el confinamiento por COVID-19 en México. Retos, 42, 1-11. https: / /doi.org/10.47197/retos.v42i0.86242

Hortigüela-Alcalá, D., Hernando Garijo, A., \& PérezPueyo, Á. (2021). La educación física en el contexto COVID-19. Un relato de profesores de diferentes etapas educativas. Retos, 41, 764-774. https:// doi.org/10.47197/retos.v41i0.86368

Jeong, H.-C., \& So, W.-Y. (2020). Difficulties of online physical education classes in middle and high school and an efficient operation plan to address them. International Journal of Environmental Research and Public Health, 17(19), 7279. https://doi.org/10.3390/

\section{ijerph17197279}

López-Fernández, I., \& Malavé, P. (2009). Las gincanas como recurso metodológico en el área de educación física. Tándem: Didáctica de la Educación Física, 31 , 110120.

López-Postigo, L., Burgueño, R., González-Fernández, F. T., \& Morente-Oria, H. (2021). El edublog en educación física como herramienta para la coeducación, el compromiso y la intención de ser físicamente activo. Espiral. Cuadernos del Profesorado, 14(28), 1 8. http: / /dx.doi.org/10.25115/ecp.v14i28.3819

Marín Díaz, V., Sampedro, B. E., Muñoz, J. M., \& Salcedo, P. (2020). El blog en la formación de los profesionales de la educación. Revista Electrónica Interuniversitaria de Formación del Profesorado, 23(2), Article 2. https: / /doi.org/10.6018/reifop.414061

Mercier, K., Centeio, E., Garn, A., Erwin, H., Marttinen, R., \& Foley, J. (2021). Physical education teachers' experiences with remote instruction during the initial phase of the COVID-19 pandemic. Journal of Teaching in Physical Education, 40(2), 1-6. https: / / doi.org/10.1123/jtpe.2020-0272

Moher, D., Liberati, A., Tetzlaff, J., Altman, D. G., \& Group, P. (2009). Preferred reporting items for systematic reviews and meta-analyses: The PRISMA statement. PLoS med, 6(7), e1000097. https:/ / doi.org/10.1371/journal.pmed.1000097

Rovira, C., Guerrero-Solé, F., \& Codina, L. (2018). Received citations as a main SEO factor of Google Scholar results ranking. Profesional de la Información, 27(3), 559-569. https://doi.org/10.3145/ epi.2018.may.09

Sierra-Díaz, J., González-Víllora, S., Toledo-Guijarro, J. A., \& Bermejo-Collada, C. (2021). Reflexiones sobre el proceso de enseñanza y aprendizaje en Educación Física durante la pandemia por COVID-19. Un caso real. Retos, 41, 866-878. https: / / doi.org/ 10.47197/retos.v41i0.85946

Varea, V., \& González-Calvo, G. (2020). Touchless classes and absent bodies: Teaching physical education in times of Covid-19. Sport, Education and Society. https: / /doi.org/10.1080/13573322.2020.1791814

Wakabayashi, D. (2018, septiembre 7). ¿Cómo funciona el algoritmo de búsqueda de Google? The New York Times. https: / / www.nytimes.com/es/2018/09/ 07/espanol/google-algoritmo-busqueda-trump.html 